\title{
Distributed delay input shaper design by optimizing smooth kernel functions
}

\author{
Dan Pilbauer ${ }^{\mathrm{a}, \mathrm{b}, *}$, Wim Michiels $^{\mathrm{a}}$, Tomáš Vyhlídal ${ }^{\mathrm{b}}$ \\ ${ }^{a}$ Department of Computer Science, Katholieke Universiteit Leuven, Celestijnenlaan 200A, \\ B-3001 Heverlee, Belgium \\ ${ }^{b}$ Department of Instrumentation and Control Eng., Faculty of Mechanical Engineering, \\ Czech Technical University in Prague, Technická 4, 16607 Praha 6, Czech Republic
}

\begin{abstract}
The aim of this paper is the development of a general class of input shapers with distributed time delay which leads to retarded spectral properties. The design of the shaper is formulated as a multi-objective optimization problem, where response time and robustness, expressed in terms of residual vibrations, are the main objectives. As a part of the optimization formulation, common requirements for input shapers such as non-decreasing step response and unity steady state gain are considered in the design. Moreover, additional optional requirements, such as smoothness of a step response, jerk and even jounce limits can be added to optimization procedure. The resulting problem can be solved using convex optimization techniques. Several illustrative examples are presented in comparison with classical input shaping techniques. Finally, implementation aspects are discussed. The paper is accompanied by an implementation in MATLAB, including a user-friendly interface for the interactive shaper design.
\end{abstract}

Keywords: input shaping, time-delay, multi-objective, convex optimization, Pólya's relaxation, Pareto front

\section{Introduction}

Since the early results by Smith [1 achieved in 1950s, the input shaping techniques have undergone an extensive development. The architecture of input shapers is shown in Fig. 1. The scheme depicts a classical feedback scheme with an input shaper $S(s)$. The input shaper has reference signal $w$ as an input and the output goes through an intermediate feedback loop to the input of the flexible structure. The goal of such a scheme is to control the plant without exciting an oscillatory mode of the flexible structure $F(s)$. The shaper $S(s)$ serves to compensate this mode. The most common types of shapers for precompensating oscillatory modes of flexible mechanical systems involve lumped

\footnotetext{
* Corresponding author

Email address: dan.pilbauer@cs.kulueven.be (Dan Pilbauer)
} 
delays in the structure. The most simple input shaper, known as the Posicast [1] or zero-vibration (ZV) shaper 2, 3, contains a single lumped delay. Involving more lumped delays in the shaper structure allows increasing the robustness in the oscillatory mode suppression, as it as been done in the zero-vibrationderivative (ZVD) shaper or extra insensitive (EI) shapers 4], see also the generalized shaper design in $[5$. Input shapers can also be targeted to suppress more than one mode, as considered for example in [6, 7]. In the discrete time-domain, the input shaping was addressed e.g. by, $7,8,8,9,10$. Various robustness issues concerning input shaping were targeted in [11, 12, 13, 14] and [15]. From a wide range of application directions, let us mention flexible manipulators and cranes [16] or a very flexible and high-friction flexible manipulator system [17. Multiinput multi-output systems using input shaping techniques were presented for twin rotor in [18, as well as for industrial robots in [19, 20].

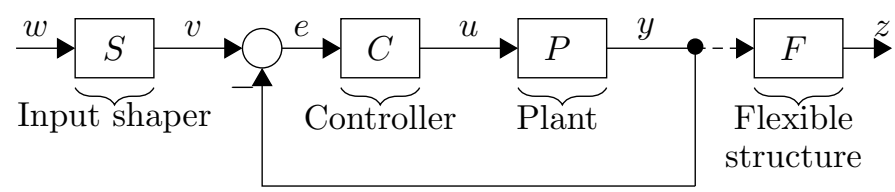

Figure 1: The classical feedforward application of input shaper with the objective to compensate the undesirable oscillatory modes of $\mathrm{F}(\mathrm{s})$.

Next to shapers with lumped delays, trapezoidal, S-curve and trigonometric input smoothers can be used for the flexible mode compensation, see e.g. 21]. However, as shown in [22], the input shaping is considerably faster and more efficient for reducing the vibrations compared to the command smoothing. As also reported in 22, these methods usually fail to fully exploit the known properties of the system such as natural frequency and damping ratio and instead simply provide a low pass filtering effect. In [23, it was demonstrated that these smoothers can be represented using distributed time delays. An alternative solution involving delays was considered by Singh in 24, where he proposed jerk limited input shapers for both single and multi-mode cases. Compared to the classical shapers, the output of the signal is smoothened by various types of filters.

The presented work is a further step in a systematic research on involving distributed delays in the structure of the shapers by the authors' team. The first results were presented in 25, 26, where the lumped delay in the ZV shaper structure was substituted by an equally distributed delay. Subsequently, more complex delay distributions were considered in [27. Next to the smoothening effect at the signal accommodation part, the retarded characteristics of the shaper spectrum can be considered as an implementation benefit, particularly, if the shaper is implemented within a closed loop system in the inverse form [28, 29. Namely, the spectrum of poles with high magnitudes tend to match the spectrum of shaper zeros. The system dynamics are then described by a delay system of retarded type, or a delay systems of neutral type if inverse shaper with lumped delays is used. A retarded spectrum has a different distribution 
of eigenvalues, i.e., at the high magnitudes, the roots of a retarded system follow the asymptotic exponential curves that depart of the imaginary axis to the left with increasing moduli 30. For neutral systems, the situation is more complicated. At least a part of the infinite spectrum is located in a strip of finite width that is parallel to the imaginary axis. Besides, the real upper bound of the neutral spectrum can be very sensitive to small changes in the delays. This leads to a requirement of so-called strong stability [31, 32, which is very restrictive from an application point of view. In the recent work 23, an optimization based technique, the constrained linear least squares method in particular, was applied to the shaper design. The work was motivated by design algorithms for digital signal shapers 33, 34, see also a recent work [35] (Chapter 6) on optimization based design of multi-mode shapers with lumped delay, and waveform command based shaping control of multi-mode systems [36. The delay distribution in 23 consisted of a discrete series of equally distributed time delays and the optimization objective was to achieve enhanced robustness of the shaper. In [37, zero phase tracking was performed by using an input shaping technique, where the design of the shaper is defined as a minimax problem. The application of a zero phase tracking input shaper on a medical $\mathrm{x}$-ray system was performed in 38 ]

The main objective of this paper is to propose novel class of shapers, characterized by a distributed delay with a smooth kernel function, and to present on optimization approach for the corresponding shapers tuning. This will be done by optimizing the shape of a selected smooth polynomial kernel function. Besides, compared to 23, additional options and constraints on the shaper properties and robustness will be considered providing the end-user extensive freedom in defining requirements on the optimized shaper performance.

Let us remark that the presented work is also related to work by Cole, et al. [39, 40, 41, where the shaper was considered and designed as a general finite impulse response (FIR) filter. The FIR filters are designed to operate on an arbitrary command input signal to ensure a finite settling time, prescribed roll-off rate and the filter frequency response. Analogously to the proposed shapers with distributed delays, the designed FIR filters produce an input smoothing effect. Next to the fact that a completely different methodology has been involved in this work, utilizing time delay system theory in combination with optimization methods, the main contribution of this paper with respect to the work by Cole, et al., consists of taking into account robustness criteria in terms of residual vibrations as well as further structural issues on the shaper design. Besides, the implementation by a time delay system with discrete delay is considered, next to the implementation by the convolution integral discretization, often applied for FIR filters.

The paper is structured as follows. A review of delay based shapers is given in Sec. 2, followed by a proposition of the novel form of input shapers. Sec. 3 describes requirements necessary for an appropriate functionality of the input shapers. In Sec. 4. an optimization problem is defined for the shaper design with constraints from Sec. 3 and solved by using standard optimization techniques. Sec. 5 shows numerous results of the proposed method. In Sec. 6 implemen- 
tation aspects are discussed and the paper concludes with final remarks in Sec. 7.

\section{Preliminaries}

\subsection{Review of delay based shapers}

The objective of applying input shapers in a serial interconnection with a system is to fully or partially compensate its oscillatory mode, which is determined by a couple of poles $\hat{s}_{z}=-\beta \pm j \Omega, \beta=\omega \zeta, \Omega=\omega \sqrt{1-\zeta^{2}}$, where $\zeta, \omega$ are the damping and natural frequency of the mode to be compensated.

The general form of input shapers involving lumped delays is given as follows

$$
u(t)=A_{0} w(t)+\sum_{k=1}^{N} A_{k} w\left(t-\tau_{k}\right),
$$

with the input $w \in \mathbb{R}$ and the output $u \in \mathbb{R}, A_{k} \in \mathbb{R}$ are the gains satisfying $\sum_{k=0}^{N} A_{k}=1$ and $\tau_{k} \in \mathbb{R}^{+}$are the delays. Considering $\tau_{1}<\tau_{2}<\ldots<\tau_{N-1}<$ $\tau_{N}$, the common requirement on nondecreasing step response results in the condition $\sum_{k=1}^{m-1} A_{k} \leq \sum_{k=1}^{m} A_{k}$, for $m=1,2, . . N$. The transfer function of the shaper is given by

$$
S(s)=A_{0}+\sum_{k=1}^{N} A_{k} e^{-s \tau_{k}} .
$$

The shaper in the form (2) has no poles, but it has infinitely many zeros forming a neutral chain in the complex plane [30, 23. In the spectral domain based synthesis of the shaper, its dominant zeros are distributed in such a way so that the undesirable oscillatory poles of the system are fully (e.g. ZV, DZV) or even robustly (e.g. EI, EEI shapers [42]) compensated. The simplest ZV shaper is characterized with $N=1$ and the parameters $A_{0}=\frac{\exp \frac{\beta}{\Omega} \pi}{1+\exp \frac{\beta}{\Omega} \pi}, \tau_{1}=\frac{\pi}{\Omega}$ and $A_{1}=1-A_{0}$. A series of two $\mathrm{ZV}$ shapers then provides the zero vibration derivative (ZVD) shaper [42] with $N=2$ and maximum delay $\tau_{2}=2 \tau_{1}$. A general form of a shaper with distributed delay can be described as

$$
u(t)=A w(t)+(1-A) \int_{0}^{T} w(t-\eta) d h(\eta) .
$$

with the parameter $A \in[0,1]$ and the delay distribution $h(\eta)$ over the finite length segment $\eta \in[0, T]$, satisfying $h(\eta)=1, \eta \geq T$. A common requirement on the delay distribution is the nondecreasing step response shape over $\eta \in[0, T]$. The transfer function of the distributed delay (3) is given by

$$
S(s)=A+(1-A) F(s, T)
$$

where $F(s, T)=L\left\{\int_{0}^{T} w(t-\eta) d h(\eta)\right\}$ is the Laplace transform of the delay. In [25, 26], a zero vibration shaper with equally distributed delay (ZVD) was 
proposed, considering $F(s)=\frac{1-e^{-s T}}{s T}$. The main benefits compared to the classical shaper was the retarded spectrum of shaper zeros, which proved useful in feedback applications of the shaper [28, [29], and the smoother accommodation and filtering effect. A negative aspect compared to the classical ZV shaper was the increased response time length. This inefficiency can partly be overcome by combining the lumped and equally distributed delay $F(s, T, \alpha)=\frac{\mathrm{e}^{-\alpha T s}-\mathrm{e}^{-T s}}{(1-\alpha) s}$ [27, where the parameter $\alpha$ determines the ratio between the lengths of the lumped part and the overall delay length $T$. An extension towards more general delay distributions, e.g. with S-shape $F(s)=\frac{4\left(1-2 e^{-s \frac{T}{2}}+e^{-s T}\right)}{T^{2} s^{2}}$ or trigonometric delay $F(s, T)=\frac{4 \pi^{2}\left(1-\mathrm{e}^{-T s}\right)}{T s\left(T^{2} s^{2}+4 \pi^{2}\right)}$ was proposed in [23], along with a fully analytical parameterization procedure. In order to increase robustness, i.e. provide distributed delay alternatives to the classical EI, ZVD shapers, a least squares approach was proposed in $\left[23\right.$, considering $F(s)=\frac{\sum_{k=0}^{N} a_{k} \mathrm{e}^{-s \tau_{k}}}{s}$. In the procedure, the delays $\tau_{k}, k=0 . . N$ covering equidistantly the interval the delay length interval $[0, T]$ are fixed and the parameters $A$ and $a_{k}, k=0 . . N$ are free parameters to optimize a residual vibration characteristic.

\subsection{Proposed novel class of input shapers}

We consider a class of input shapers, of the form (3), described by

$$
u(t)=A w(t)+\int_{0}^{T} g(\theta) w(t-\theta) d \theta,
$$

where $w(t) \in \mathbb{R}, u(t) \in \mathbb{R}$ are input and output, respectively, the smooth function $g$ is the general kernel function of the distributed delay, $T>0$ is the maximum time-delay and $A \in[0,1]$ is the static gain. The transfer function of the shaper is given by

$$
G(s)=A+\int_{0}^{T} g(\theta) e^{-s \theta} d \theta .
$$

The kernel function can be expressed as a combination of various types of basis functions (e.g. splines, exponentials, polynomials,...). Since implementation and realization constraints need to be taken into an account when designing the kernel function, this work is based on the kernel function $g$ chosen as the polynomial

$$
g(\theta)=\sum_{i=0}^{N_{p}} a_{i} \theta^{i}
$$

where $a_{i} \in \mathbb{R}, i=0,1, \ldots, N_{p}$, are the coefficients and $N_{p}$ is the selected degree of the polynomial. Thus, the transfer function (6) can be rewritten into a form

$$
G(s)=A+\sum_{i=0}^{N_{p}} a_{i} g_{i}(s),
$$


where the functions $g_{i}(s)$ are given by

$$
g_{i}(s)=\int_{0}^{T} \theta^{i} e^{-s \theta} d \theta=\frac{i !-\sum_{j=0}^{i} \frac{i !}{(i-j) !}(T s)^{i-j} e^{-T s}}{s^{i+1}},
$$

and can be interpreted as so called "moments" of $e^{-s \theta}$.

The impulse response of the input shaper (5) and (7) is given by

$$
h(t)= \begin{cases}A \delta(t)+g(t), & t \in[0, T], \\ 0, & t \geq T,\end{cases}
$$

with $\delta$ denoting the Dirac impulse, and the step response by

$$
s(t)= \begin{cases}A+\int_{0}^{t} g(\theta) d \theta, & t \in[0, T], \\ 1, & t \geq T .\end{cases}
$$

The spectrum of zeros is given as the solution of the equation $G(s)=0$. Multiplying both sides of the equation by $s^{N_{p}+1}$, we obtain the following equation

$$
A s^{N_{p}+1}+\sum_{i=0}^{N_{p}} a_{i}\left(i ! s^{N_{p}-i}-\sum_{j=0}^{i} \frac{i !}{(i-j) !} T^{i-j} s^{N_{p}-j} e^{-T s}\right)=0
$$

which has the same distribution of zeros as $G(s)$, except for additional zeros in the $s$-plane origin.

The inverse application of the shaper in a feedback interconnection requires $A>0$. It is easy to see that for $A>0$, the quasi-polynomial at the left-hand side of $(10)$ is retarded, because of the fact that the $s$ power corresponding to the exponential terms ranges from 0 (for $i=N_{p}$ ) to $N_{p}$ (for $i=0$ ), i.e. it is lower than the quasi-polynomial order $N_{p}+1$. This is a desirable property since in such a feedback configuration the shaper zeros are turned into poles, see [28, 29].

For $A=0$, which is the case considered as well in what follows, the spectrum can be either retarded or neutral, depending on the values of the parameters $a_{i}, i=1 . . N_{p}$ and $T$. It needs to be stressed that in the feed-forward shapersystem interconnection the retarded character of the spectrum is not required and shapers with neutral spectrum of zeros can be applied too.

\section{Requirements on the shaper functionality}

In this section we outline various design requirements for input shapers that can be taken into account in their design by optimization methods. Most of them will be directly expressed in terms of linear equality or inequality constraints on the shaper parameters $A, a_{0}, \ldots, a_{N_{p}}$. Others will be expressed by the nonnegativity of the polynomial on an interval. 


\subsection{Linear equality constraints}

The first linear constraint stems from placing zeros of the shaper at the expected position of the oscillatory mode to be compensated,

$$
\hat{s}_{n}=-\zeta_{\text {nom }} \omega_{\text {nom }} \pm j \sqrt{1-\zeta_{\text {nom }}^{2}} \omega_{\text {nom }}
$$

where $\omega_{\text {nom }}$ and $\zeta_{\text {nom }}$ are the nominal frequency and damping. By placing zeros of the shaper, we achieve zero-pole cancellation, which removes entirely the undesired oscillatory modes. These requirements corresponds to

$$
G\left(\hat{s}_{n}\right)=0 \Rightarrow A+\sum_{i=0}^{N_{p}} a_{i} g_{i}\left(\hat{s}_{z}\right)=0,
$$

which can be turned into two real equations for the case of a complex zero,

$$
\begin{aligned}
& \Re\left\{G\left(\hat{s}_{z}\right)\right\}=0, \\
& \Im\left\{G\left(\hat{s}_{z}\right)\right\}=0 .
\end{aligned}
$$

It is also possible to place more zeros. However, each additional zero decreases the number of degrees of freedom. In order to arrive at a feasible solution, this may lead to a significant increase of the time delay of the shaper.

The second equality constraint comes from the basic requirement on input shaping to have a static gain equal to one, which leads to

$$
G(0)=1 \Rightarrow A+\sum_{i=0}^{N_{p}} a_{i} g_{i}(0)=1 .
$$

The additional linear equality constraint that might be required corresponds to the requirement of continuity of both the step response and its derivatives at times $t=0$ or (and) $t=T$. At $t=0$ it is expressed by

$$
A=0, \quad g(0)=a_{0}=0,
$$

and for $t=T$ by

$$
g(T)=\sum_{i=0}^{N_{p}} a_{i} T^{i}=0 .
$$

\subsection{Linear inequality constraints}

The first constraints come from the basic requirement on the delay free part of the shaper $0 \leq A \leq 1$.

The next fundamental requirement that need to be considered is the nondecreasing step response, or equivalently, the non-negative impulse response, which can be formulated as

$$
g(\alpha) \geq 0, \forall \alpha \in[0, T] .
$$


Condition (17) is a semi-infinite polynomial inequality (requirement to be satisfied for a continuum of $\alpha$ values). The problem can be solved via Pólya's relaxations, which will be addressed in the following subsection.

As demonstrated and motivated by Singh [24, limiting the jerk, defined as the $1^{\text {st }}$ derivative of impulse response, can help to increase durability of actuators in the control systems. Analogously, limiting the jounce - the $2^{\text {nd }}$ derivative of the impulse signal can also be imposed. For example as demonstrated in [43], [44, limiting jerk and jounce reduces CNC machining vibrations and increases product quality. These quantities have also been taken into account in planning a trajectory of a manipulating robot in [45] or [46. The constraints on the jerk and jounce are described by

$$
\left\{\begin{array}{l}
|\dot{g}(\alpha)| \leq J_{1} \\
|\ddot{g}(\alpha)| \leq J_{2}
\end{array}, \forall \alpha \in[0, T]\right.
$$

where $J_{1,2}$ are chosen limits on jerk and jounce, respectively. The inequality constraints (18) are also semi-infinite. They can be transformed to the following polynomial inequalities,

$$
\left\{\begin{array}{l}
\dot{g}(\alpha)+J_{1} \geq 0 \\
J_{1}-\dot{g}(\alpha) \geq 0 \\
\ddot{g}(\alpha)+J_{1} \geq 0 \\
J_{1}-\ddot{g}(\alpha) \geq 0
\end{array}, \forall \alpha \in[0, T],\right.
$$

and included via Pólya's relaxation technique, which is addressed next.

\subsection{Pólya's relaxation}

When applying Pólya's relaxation to the polynomial inequality

$$
p(\alpha) \geq 0, \forall \alpha \in[0, T]
$$

with $p$ denoting a polynomial of degree $N_{p}$, the following steps need to be followed [4], where the first two reformulate the polynomial $p$ to a homogeneous polynomial over a unit simplex:

1. Rescale the interval for $\alpha$ to $[0,1]$ by introducing a new variable $\theta=\frac{\alpha}{T} \Rightarrow$ $\theta \in[0,1]$.

2. Set $\theta=\theta_{1}$, introduce the additional variable $\theta_{2} \in \mathbb{R}$, and homogenize the polynomial $p\left(T \theta_{1}\right)$ by multiplying single monomials with powers of $\left(\theta_{1}+\theta_{2}\right)$, until all monomials have the same degree $N$ (larger than or equal to $N_{p}$ ). The non-negativity requirement 200 on $p$ is now equivalent with the non-negativity requirement of the corresponding homogeneous multivariate polynomial $p_{N}\left(\theta_{1}, \theta_{2}\right)$ for all $\theta_{1}$ and $\theta_{2}$ satisfying

$$
\theta_{1} \geq 0, \theta_{2} \geq 0, \theta_{1}+\theta_{2}=1
$$

i.e., for all $\left(\theta_{1} \theta_{2}\right)$ belonging to the unit simplex in $\mathbb{R}^{2}$. 
3. Compute the coefficients of the multi-variate polynomial $p_{N}\left(\theta_{1}, \theta_{2}\right)$.

4. Sufficient conditions for (20) are obtained by requiring that all coefficients of $p_{N}$ be non-negative.

5. If necessary, increase Pólya's relaxation degree $N$ and repeat from step (2) on. It can be shown that the gap between sufficient and necessary conditions tends to zero by increasing Pólya's relaxation degree, see, e.g., 48.

Example. We consider requirement (17) of a non-decreasing step response on interval $[0, T]$, for the case where $N_{p}=2$, i.e.,

$$
g(\alpha):=a_{0}+a_{1} \alpha+a_{2} \alpha^{2} \geq 0, \forall \alpha \in[0, T] .
$$

With $\theta=\frac{\alpha}{T}$, condition $22 p$ is equivalent to

$$
a_{0}+a_{1} T \theta+a_{2} T^{2} \theta^{2} \geq 0, \forall \theta \in[0,1] .
$$

This in turn is equivalent to

$$
\begin{aligned}
& a_{0}\left(\theta_{1}+\theta_{2}\right)^{2}+a_{1} T\left(\theta_{1}+\theta_{2}\right) \theta_{1}+a_{2} T^{2} \theta_{1}^{2} \geq 0, \\
& \forall \theta_{1} \geq 0, \theta_{2} \geq 0: \theta_{1}+\theta_{2}=1,
\end{aligned}
$$

i.e., a positivity constraint of a homogeneous multivariate polynomial over the unit simplex. Working out this expression in monomials gives

$$
\begin{aligned}
& \theta_{1}^{2}\left(a_{0}+a_{1} T+a_{2} T^{2}\right)+\left(2 a_{0}+a_{1} T\right) \theta_{1} \theta_{2}+a_{0} \theta_{2}^{2} \geq 0, \\
& \forall \theta_{1} \geq 0, \theta_{2} \geq 0: \theta_{1}+\theta_{2}=1 .
\end{aligned}
$$

Hence, sufficient conditions for 22 are given by the linear inequalities

$$
a_{0}+a_{1} T+a_{2} T^{2} \geq 0,2 a_{0}+a_{1} T \geq 0, a_{0} \geq 0 .
$$

This is called Pólya's relaxation of degree 2. Tighter conditions can be obtained by increasing the degree of the relaxation, at the price of an increase in the number of inequalities. For Pólya's relaxation of order 3, one writes the constraint as

$$
\begin{aligned}
& a_{0}\left(\theta_{1}+\theta_{2}\right)^{3}+a_{1} T\left(\theta_{1}+\theta_{2}\right)^{2} \theta_{1}+a_{2} T^{2}\left(\theta_{1}+\theta_{2}\right) \theta_{1}^{2} \geq 0, \\
& \forall \theta_{1} \geq, \theta_{2} \geq 0: \theta_{1}+\theta_{2}=1 .
\end{aligned}
$$

Working out this expressions and requiring the coefficients of all (third-order) monomials to be non-negative results in

$$
\begin{aligned}
& a_{0}+a_{1} T+a_{2} T \geq 0,3 a_{0}+2 a_{1} T+3 a_{2} T^{2} \geq 0, \\
& 3 a_{0}+a_{1} T \geq 0, a_{0} \geq 0 .
\end{aligned}
$$

\section{Optimization problem formulation}

We present a procedure for the design of the shaper parameters $A, a_{0}, \cdots, a_{N_{p}}$ and $T$. The procedure is based on a multi-objective optimization problem yielding a trade-off between a fast response time of the shaper and a robustness requirement, expressed in terms of residual vibrations. The optimization problem is constrained by selected requirements specified in Section 3 


\subsection{Residual vibrations}

The design procedure takes into account robustness of the shaper, in the sense that it is less insensitive with respect to changes of the suppressed system's parameters. Our robustness criterion is expressed in terms of the residual vibrations introduced by [4], which can be expressed in terms of the transfer function (6), as shown in 23.

$$
V(\zeta, \omega)=\left|G\left(-\omega \zeta-j \omega \sqrt{1-\zeta^{2}}\right)\right| e^{\zeta \omega T},
$$

and which takes into account uncertainty not only in the nominal frequency (as it is usual), but also in the damping of the vibration to be suppressed. Formula (24) expresses the amplitude of the residual vibration at time $t=T$.

More precisely, let $s=-\omega \zeta-j \omega \sqrt{1-\zeta^{2}}$ be the pole to be compensated by a shaper zero and assume that $\omega \in I, \zeta \in I_{2}$, where

$$
I_{1}=\left[\omega_{\min }, \omega_{\max }\right], I_{2}=\left[\zeta_{\min }, \zeta_{\max }\right],
$$

with a nominal frequency $\omega_{\text {nom }}$ and a nominal damping $\zeta_{\text {nom }}$ assumed to be the midpoints of these intervals.

To handle the uncertainty in $\omega$ and $\zeta$, we define a grid of $N_{\omega}$ and $N_{\zeta}$ Chebyshev points, which are more efficient for polynomial approximation [50], on $I_{1}$ and $I_{2}$

$$
\begin{aligned}
\omega_{k} & =\left(\frac{\omega_{\max }+\omega_{\min }}{2}\right)-\left(\frac{\omega_{\max }-\omega_{\min }}{2}\right) \cos \left(\frac{(k-1) \pi}{N_{\omega}-1}\right) ; k=1, \ldots, N_{\omega}, \\
\zeta_{l} & =\left(\frac{\zeta_{\max }+\zeta_{\min }}{2}\right)-\left(\frac{\zeta_{\max }-\zeta_{\min }}{2}\right) \cos \left(\frac{(k-1) \pi}{N_{\zeta}-1}\right) ; l=1, \ldots, N_{\zeta} .
\end{aligned}
$$

Define the vector of gains to be assessed $\mathbf{x}=\left[\begin{array}{lllll}A & a_{0} & a_{1} & \cdots & a_{N_{p}}\end{array}\right]^{\top}$, the robustness criterion can be expressed by

$$
f(\mathbf{x}, T)=\frac{1}{N_{\omega} N_{\zeta}} \sum_{k=1}^{N_{\omega}} \sum_{l=1}^{N_{\zeta}} V\left(\zeta_{l}, \omega_{k}\right)^{2} .
$$

The shaper transfer function (6) can be expressed as

$$
G\left(-\omega \zeta-j \omega \sqrt{1-\zeta^{2}}\right)=L(\zeta, \omega, T) \mathbf{x}
$$

with

$$
L(\zeta, \omega, T)=\left[1 g_{0}\left(-\omega \zeta-j \omega \sqrt{1-\zeta^{2}}\right) \cdots g_{N_{p}}\left(-\omega \zeta-j \omega \sqrt{1-\zeta^{2}}\right)\right] .
$$

Consequently we have

$$
\begin{aligned}
V(\zeta, \omega)^{2} & =\mathbf{x}^{\top} L(\zeta, \omega, T) L(\zeta, \omega, T) \mathbf{x} e^{2 \zeta \omega T} \\
& =\mathbf{x}^{\top} \Re(L(\zeta, \omega, T) L(\zeta, \omega, T)) \mathbf{x} e^{2 \zeta \omega T}
\end{aligned}
$$


and the robustness measure takes the quadratic form

$$
f(\mathbf{x}, T)=\mathbf{x}^{\top} H(T) \mathbf{x}
$$

where

$$
H(T)=\frac{1}{N_{\omega} N_{\zeta}} \sum_{k=0}^{N_{\omega}} \sum_{l=0}^{N_{\zeta}} e^{2 \zeta_{l} \omega_{k} T} \Re\left(L\left(\zeta_{l}, \omega_{k}, T\right) L\left(\zeta_{l}, \omega_{k}, T\right)\right) .
$$

Note that matrix $H(T)$ is postive semi-definite for every $T$, hence, the quadratic form $(28)$ is a convex function.

\subsection{A multi-objective optimization problem for the shaper design}

Recall that the shaper requirements in Section 3 are all expressed in, or transformed to, linear equality and inequality constraints in the coefficients $\left(A, a_{0}, \ldots a_{N p}\right)$. Hence, the selected constraints can be compactly written as

$$
\left\{\begin{array}{l}
A_{1}(T) \mathbf{x} \geq b_{1}, \\
A_{2}(T) \mathbf{x}=b_{2},
\end{array}\right.
$$

with the notation $A_{1}(T), A_{2}(T)$ the dependence of the selected (in)equality constraints on $T$ is stressed (in fact, the dependence on $T$ is polynomial).

A practical shaper design is characterized by a compromise between two main conflicting requirements: a fast response time $T$ on the one hand, and a small size of the residual vibrations close to the targeted mode, on the other hand. For a given parameter $\alpha \in[0,1]$ this brings us the optimization problem

$$
\begin{aligned}
& \min _{T, \mathbf{x}} \alpha\left(\frac{T}{T_{\mathrm{nom}}}\right)^{2}+(1-\alpha) \frac{\mathbf{x}^{\boldsymbol{\top}} H(T) \mathbf{x}}{R_{\mathrm{nom}}^{2}}, \\
& \text { subject to } \\
& \left\{\begin{array}{l}
A_{1}(T) \mathbf{x} \geq b_{1}(T), \\
A_{2}(T) \mathbf{x}=b_{2}(T) .
\end{array}\right.
\end{aligned}
$$

The scale factor $T_{\text {nom }}$ is included in the objective function since the response time of the shaper should be considered in a relative sense, in comparison to the nominal period time of the oscillation to be suppressed. The scale factor $R_{\text {nom }}$ is the reference for the residual vibrations. The scale factor is taken as $R_{\text {nom }}=0.05$ since $5 \%$ of the residual vibrations is considered as a limit for a robustness.

The optimization problem (31) is a nonlinear programing problem and convergence is not guaranteed. However, we can exploit that for a fixed $T$ it can be solved as a convex quadratic problem (QP) because of $H(T) \geq 0$ and the linearity of the constraints, of which the global optimum can be found using standard optimization method for convex optimization. Here, the optimization is solved by the tailored interior-point algorithm implemented in the form of a MATLAB function. In addition, for fixed $T$ the optimal $\mathbf{x}$ does not depend on alpha. Therefore, the solution of problem (31) can be obtained by one sweep over scalar 
parameter $T$, and solving the corresponding QPs. Note that the existence of the solution is only limited by constraints 30 , and if selected too restrictive the solution might be infeasible. Finally, by varying parameter $\alpha$ from zero to one, the full Pareto front is generated of the multi-objective optimization problem

$$
\begin{aligned}
& \min _{T, \mathbf{x}}\left\{\left(\frac{T}{T_{\text {nom }}}\right)^{2}, \frac{\mathbf{x}^{\boldsymbol{\top}} H(T) \mathbf{x}}{R_{\text {nom }}^{2}}\right\}, \\
& \text { subject to } 30 .
\end{aligned}
$$

As we shall see, generating a full Pareto front, which the accompanying software does, is very helpful for the user to find an appropriate trade-off between the two objective, in accordance with the application in hand.

\subsection{Design procedure}

The constraints for the optimization presented in the $\$ 4.2$ can be divided into two groups. The first group consists of conditions that always have to be included in the optimization procedure. These constraints are:

- Non-decreasing step response imposed via Pólya's relaxation;

- Steady state gain equal to one.

The second group consists of constraints that are not necessarily needed in the optimization procedure. Including any of these constraints leads to additional attributes of the shaper, at the price reducing the set of feasible solutions. The optional constraints include:

- Initial step gain $A$;

- Placing zeros corresponding to poles of undesirable modes;

- Continuity of the step response and its derivative;

- Jerk and jounce limits via Pólya's relaxation.

Consequently, for a given selection of constraints, a point on the Pareto front of (32) is obtained as a result of solving the optimization problem (31), where a selection of the parameter $\alpha$ results into a compromise between the response time $T$ and average residual vibrations on the domain $(\omega, \xi) \in I_{1} \times I_{2}$.

We conclude the section by pointing out the following scaling property of the optimization problem. Suppose optimization problem (31) has been solved for system parameters

$$
\zeta_{\text {nom }}, \omega_{\text {nom }},\left[\omega_{\min }, \omega_{\max }\right],\left[\zeta_{\min }, \zeta_{\max }\right], J_{1}, J_{2}
$$

yielding shaper parameters $\left(A, a_{0}, a_{1}, a_{2}, \ldots, a_{N_{p}}, T\right)$, then the solution of (31) for system parameters

$$
\zeta_{\text {nom }}, \rho \omega_{\text {nom }},\left[\rho \omega_{\min }, \rho \omega_{\max }\right],\left[\zeta_{\min }, \zeta_{\max }\right], \frac{J_{1}}{\rho}, \frac{J_{2}}{\rho^{2}},
$$


with $\rho>0$ a scaling parameter, is given by

$$
\left(A, a_{0} \rho, a_{1} \rho^{2}, a_{2} \rho^{3}, \ldots, a_{N_{p}} \rho^{N_{p}+1}, \frac{T}{\rho}\right) .
$$

A user-friendly, MATLAB based, interface for the shaper design is available from

http://twr.cs.kuleuven.be/research/software/delay-control/shaper-design/

\subsection{Note on the optimization problem}

The goal of the problem (32) is to find a set of Pareto optimal solutions and trade of between different objectives [51. The underlying assumption is that one solution from the set must be selected by the decision maker. A multi-objective optimization problem is in general defined as

$$
\min _{x} f_{1}(x), f_{2}(x), \ldots, f_{k}(x)
$$

where $k \geq 2$ and $f_{k}(x)$ is $k$-th objective function. The optimization problem (32) is in our case bi-objective and can be translated to a single objective optimization problem by scalarizing the problem as in (31), such that optimal solutions to the single-objective optimization problem are Pareto optimal solutions to the multi-objective optimization problem [52. As mentioned before we can exploit the convexity of $\sqrt{32}$ for a fixed value of $T$.

This guarantees that if a local minimum exists, then it is a global minimum 53. The existence of solution is only restricted to the set of constraints, here (30). Obviously, if the constraints are too restrictive and mutually contradictory, there might not be points that satisfy all the constraints and thus the feasible set is empty. In such case the problem has no solution [53].

\section{Results}

This section provides six design examples of shaper (3). The shapers are tuned for an oscillatory mode defined by the damping ratio $\zeta_{\text {nom }}=0.01$ and natural frequency $\omega_{\text {nom }}=1$. The uncertainty intervals are given by $I_{1}=$ $[0.85,1.15] \omega_{\text {nom }}$ and $I_{2}=[0.85,1.15] \zeta_{\text {nom }}$, and $N_{\omega}=10, N_{\zeta}=10$ number of points are used in the discretization of the $\omega$ and $\zeta$ interval, respectively.

The first three examples illustrate the design of robust shapers with different constraints. The next three examples show a comparison with the ZV shaper. The selected constraints for the examples are indicated in Table 1. The parameters of the designed shapers are shown in Table 2, where the two objectives, i.e., the response time and average residual vibrations, are shown as well.

Fig. 2 shows the complete Pareto front of multi-objective optimization problem (32) for example \#2 with constraints listed in Table1. As expected, a faster response time (horizontal axis) is obtained by increasing $\alpha$, at the price of an increase of the average residual vibrations (vertical axis) on the given intervals (25). To obtain comparable results, the parameter $\alpha$ for examples \#1-3 is selected as $\alpha=0.0501$. 


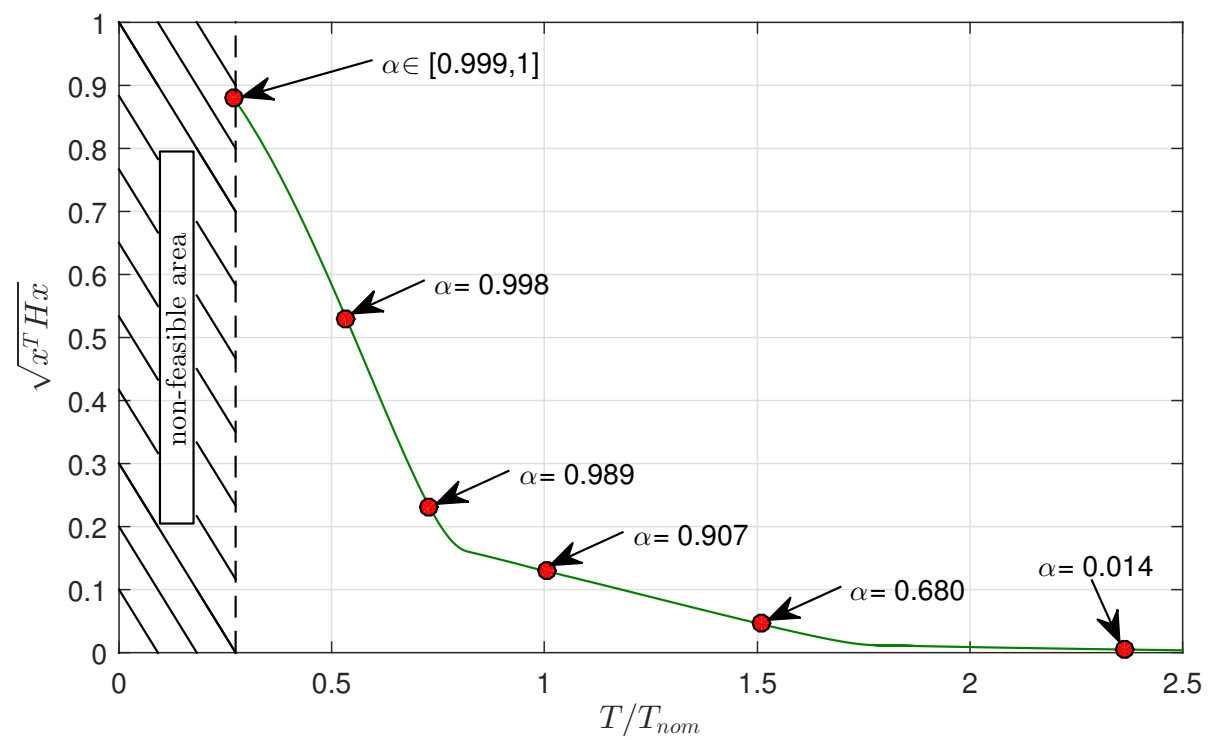

Figure 2: The Pareto front (green curve) of 32 for the first example in Section 5 The red circles represent the solutions of 31 for certain values of $\alpha$.

A comparison of step and impulse response is in Fig. 3. As expected, a smoother response leads to a (slight) increase in the response time. Step response of the interconnection with system with defined oscillatory mode is depicted in Fig. 4. Figure has highlighted 5\% range of oscillations with black dotted lines. As can be seen, the example \#3(yellowish) has no oscillations after the shaper response time $t>T$, because one of the constraints is to place an exact zero. Examples \#1 and \#2 have residual vibrations after the response time, but in a very small range, which corresponds with Pareto front charts. Next, Fig. 5 shows residual vibrations with respect to a frequency. The interval of frequencies, where the shapers are optimized, is indicated with black dashed lines around the nominal frequency $\omega_{\text {nom }}$. Shapers are also optimized in the neighbourhood of the nominal damping ratio $\zeta_{\text {nom }}$. Residual vibrations with respect to $\zeta$ and $\omega$ around the nominal values are shown in Fig 6 . Nominal values are indicated with balck dashed lines. The dependency on $\zeta$ is much small than the one on $\omega$ and almost not noticeable. A good conception about the dynamics of the shaper is provided in Fig. 7. The spectra are computed by the QPmR algorithm [54. As can be seen, the spectrum of zeros with $A>1$ in $\# 1$ is truly retarded, as derived in the Subsection 2.2. Interestingly, the spectrum corresponding to $\# 3$ is also retarded despite $A=0$, whereas the spectrum for the case \#2 is neutral. In a detailed view, the distribution of the zeros of the shapers corresponds to Fig. 5, where a decrease of residual vibrations is seen on frequencies around the zeros in the complex plane.

Examples \#4-6 are shown to provide a comparison with the classical ZV shaper, which is described by (3) for $N=1$ and where the parameters $\left(A_{0}, A_{1}\right.$ 


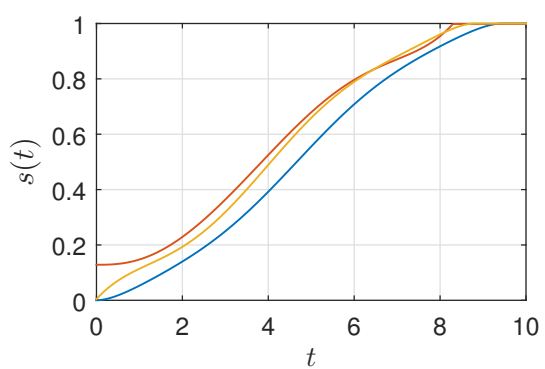

(a) Step responses

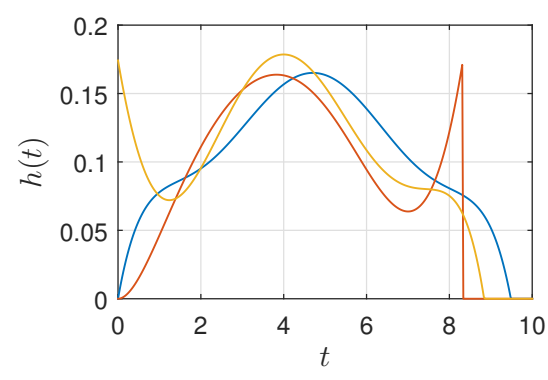

(b) Impulse responses

Figure 3: Step and impulse responses of the shaper examples \#1(red),\#2(blue) and \#3(yellowish)

and $\left.\tau_{1}\right)$ are determined by placing a zero at the oscillatory mode $\left(\zeta_{\text {nom }}=0.01\right.$, $\left.\omega_{\text {nom }}=1\right) . \quad$ To have comparative results, parameter $\alpha$ in $(31)$ is selected as $\alpha=1$, so the optimization objective insists on a response time as fast as possible. The suppression of the undesired oscillation is then imposed by placing an exact zero at $\hat{s}_{n}$. Example \#4 shows only a $10 \%$ slower response time then the $\mathrm{ZV}$ shaper and the responses are comparable. Hence, if the response time is the main criterion, the proposed shaper mimics a ZV shaper. The next two examples have more requirements on smoothness, and the response time increases accordingly. As in the previous three examples, the step response is in Fig. 8 with a comparison with the classical ZV shaper and the step response for shaper interconnected with oscillatory system as shown in Fig. 9. The residual vibrations in Fig. 10 have almost the same shape for $\omega<1$. Examples with longer response time $T$ have lower residual vibrations on higher frequencies.

\begin{tabular}{c|c|c|c|c|c}
\hline$\#$ & $\begin{array}{l}\text { placing a zero } \\
\text { on } \omega_{\text {nom }}\end{array}$ & $\begin{array}{l}\text { constraint on initial } \\
\text { step at } t=0(A=0)\end{array}$ & $\begin{array}{l}\text { zero derivative of step } \\
\text { response at } t=0\end{array}$ & $\begin{array}{l}\text { zero derivative of step } \\
\text { response at } t=T\end{array}$ & $\alpha$ \\
\hline \hline 1 & & & & & \\
\hline 2 & & $\times$ & $\times$ & $\times$ & 0.680 \\
\hline 3 & $\times$ & $\times$ & & & 0.680 \\
\hline \hline 4 & $\times$ & & & $\times$ & 0.680 \\
\hline 5 & $\times$ & $\times$ & $\times$ & $\times$ & 1 \\
\hline 6 & $\times$ & $\times$ & & & 1 \\
\hline
\end{tabular}

Table 1: Optional constraints selected for every example are indicated by $\times$. In all cases we use a polynomial of degree 7. All examples include constraints of a non-decreasing step response imposed via a Pólya's relaxation of degree 10, and of a steady state gain equal to one

\section{Implementation aspects}

The proposed form of the input shapers (5)-(10) cannot be directly implemented using standard block in a computer controlled system, hence this section 


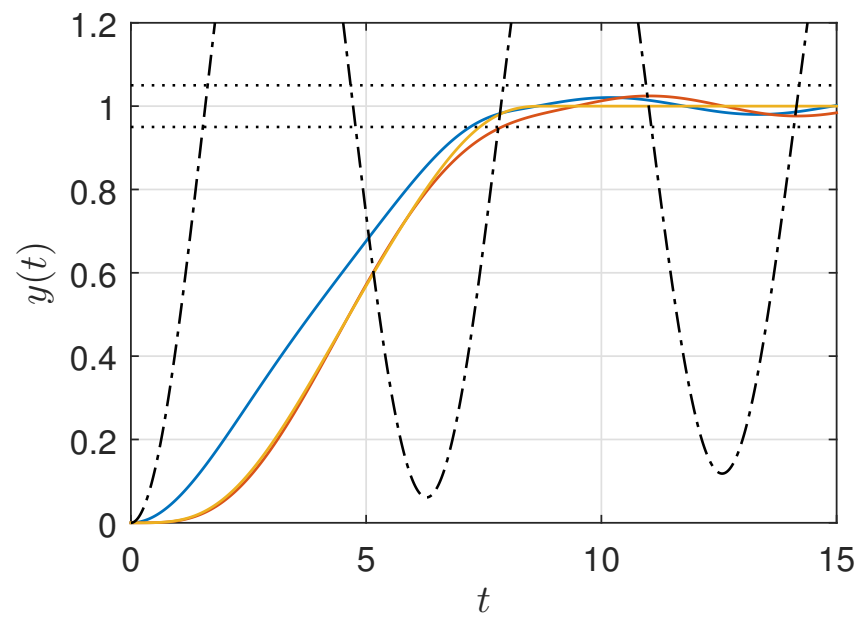

Figure 4: Step response of the shaper interconnected with a second order oscillatory system with output y for examples \#1(red),\#2(blue) and \#3(yellowish). The dot-dashed line is a system without shaper. The black dotted lines show $5 \%$ region around target value

\begin{tabular}{l|l|l|lllllllll}
\hline$\#$ & $\mathrm{~T}$ & $\sqrt{\mathbf{x}^{\top} H \mathbf{x}}$ & $\mathrm{A}$ & $a_{0}$ & $a_{1}$ & $a_{2}$ & $a_{3}$ & $a_{4}$ & $a_{5}$ & $a_{6}$ & $a_{7}$ \\
\hline \hline 1 & $2.65 \pi$ & 0.036 & 0.129 & $1.006 \mathrm{e}-7$ & $-9.954 \mathrm{e}-8$ & $7.898 \mathrm{e}-2$ & $-4.245 \mathrm{e}-2$ & $1.150 \mathrm{e}-2$ & $-1.824 \mathrm{e}-3$ & $1.515 \mathrm{e}-4$ & $-4.881 \mathrm{e}-6$ \\
\hline 2 & $3.02 \pi$ & 0.051 & 0 & 0 & 0.173 & -0.146 & $6.268 \mathrm{e}-2$ & -1.286 & $1.234 \mathrm{e}-3$ & $-4.658 \mathrm{e}-5$ & $1.850 \mathrm{e}-7$ \\
\hline 3 & $2.81 \pi$ & 0.048 & 0 & 0.175 & -0.178 & $8.062 \mathrm{e}-2$ & $3.668 \mathrm{e}-3$ & $-6.623 \mathrm{e}-3$ & $1.117 \mathrm{e}-3$ & $-6.343 \mathrm{e}-5$ & $7.584 \mathrm{e}-7$ \\
\hline \hline 4 & $1.11 \pi$ & 0.18 & 0.489 & $3.039 \mathrm{e}-5$ & $-4.788 \mathrm{e}-5$ & $2.151 \mathrm{e}-5$ & $4.822 \mathrm{e}-2$ & $-7.648 \mathrm{e}-2$ & $5.017 \mathrm{e}-2$ & $-1.602 \mathrm{e}-2$ & $2.169 \mathrm{e}-3$ \\
\hline 5 & $1.27 \pi$ & 0.17 & 0.455 & $6.709 \mathrm{e}-8$ & $2.812 \mathrm{e}-6$ & 0.181 & -0.317 & 0.239 & $-9.702 \mathrm{e}-2$ & $2.234 \mathrm{e}-2$ & $-2.187 \mathrm{e}-3$ \\
\hline 6 & $1.67 \pi$ & 0.16 & 0 & 0 & 1.253 & -1.624 & 0.893 & -0.264 & $4.316 \mathrm{e}-2$ & $-3.304 \mathrm{e}-3$ & $5.908 \mathrm{e}-5$ \\
\hline
\end{tabular}

Table 2: The optimized coefficients of the polynomial (7). The numbers in the first column correspond with example numbers. The second column is the response time of the shaper. The third column is the average of the residual vibrations.

propose two ways how to implement the shaper (5). The first method is based on an on-line computation of the integral. The second approach consists of realizing the shaper by a dynamic system. In order to avoid an unstable realization, we propose a modification of the basis functions $g_{i}$ for the second case.

\subsection{On-line computation of integral}

This method doesn't require further modifications of the shaper equation but requires the on-line evaluation of the integral in every discrete step time, which may lead to high memory and computation costs. This methods may also lead to instability when the numerical integration method is not selected appropriately (see [55] and the references therein). The shaper can be implemented as

$$
y(t) \approx A u(t)+\sum w_{k} g\left(\theta_{k}\right) u\left(t-\theta_{k}\right),
$$

where $\theta_{k}$ and $w_{k}$ are nodes and weights of the quadrature formula. 


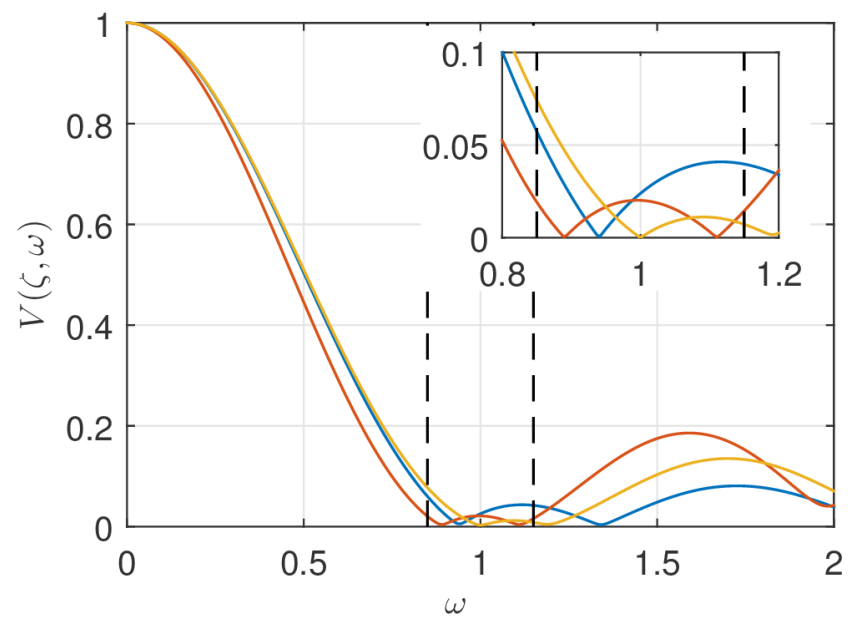

Figure 5: Residual vibrations of the shaper examples \#1(red),\#2(blue) and \#3(yellowish). The graph is plotted for nominal damping ratio $\zeta_{\text {nom }}$. The cropped zoom view shows details around nominal frequency $\omega_{\text {nom }}$.
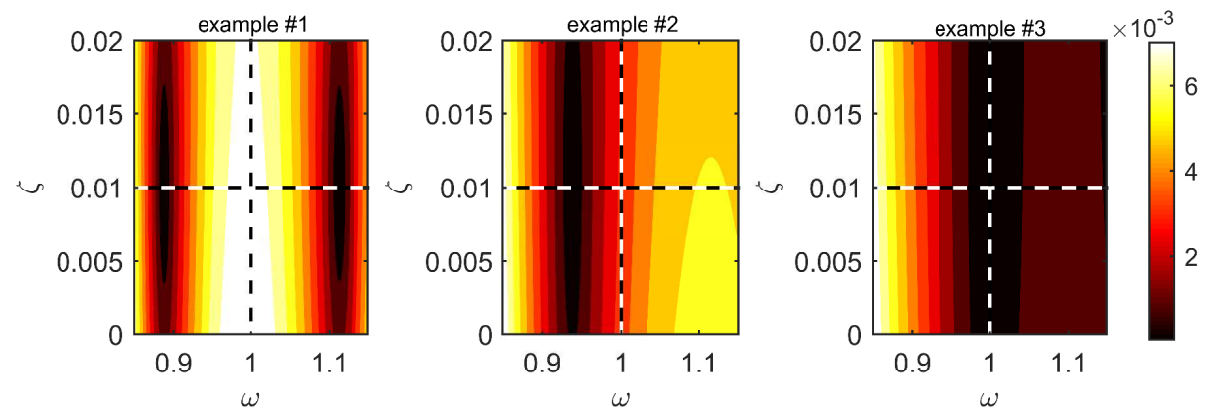

Figure 6: Contour plots of the residual vibrations of the shaper for examples \#1-\#3, with respect to $\zeta$ and $\omega$. 


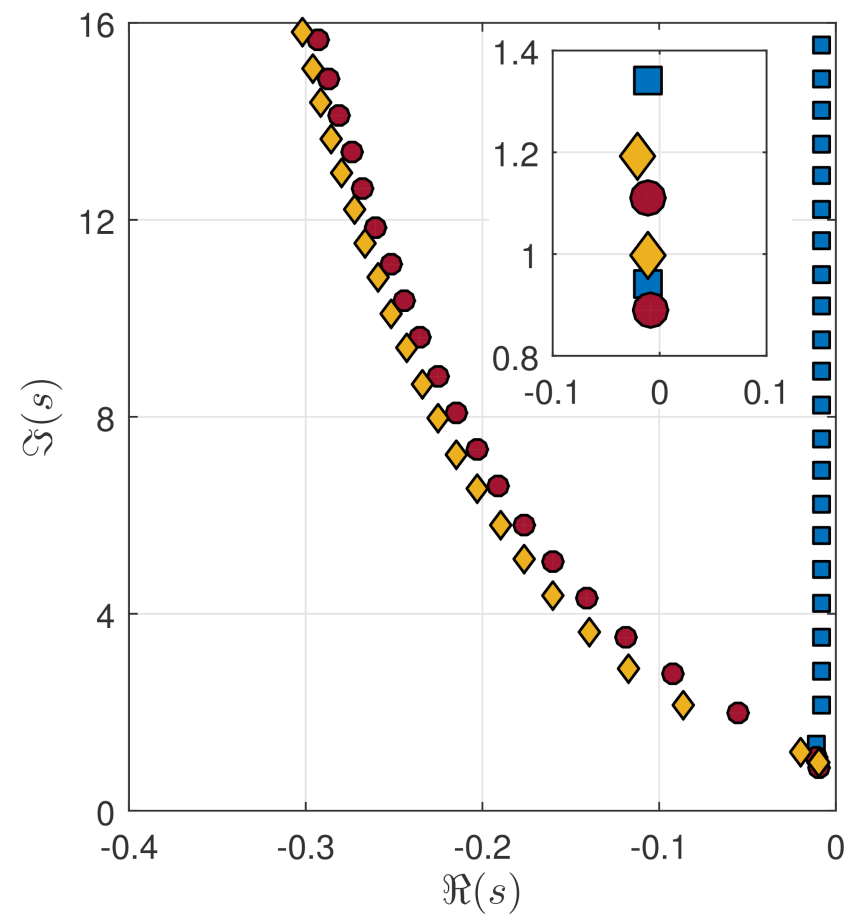

Figure 7: The zeros of the shaper with parameters given in Table 2 The spectra correspond to examples \#1(red, circles), \#2(blue, squares) and \#3(yellowish, diamonds)

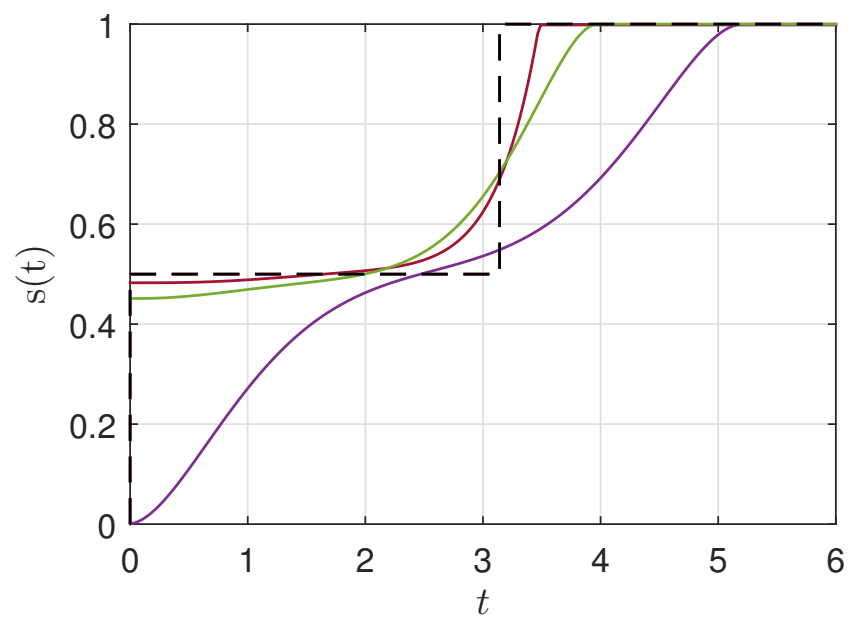

Figure 8: Step response of input shaper examples \#4(brown),\#5(green) and \#6(purple). For comparison, the ZV shaper step response shown with black dashed line 


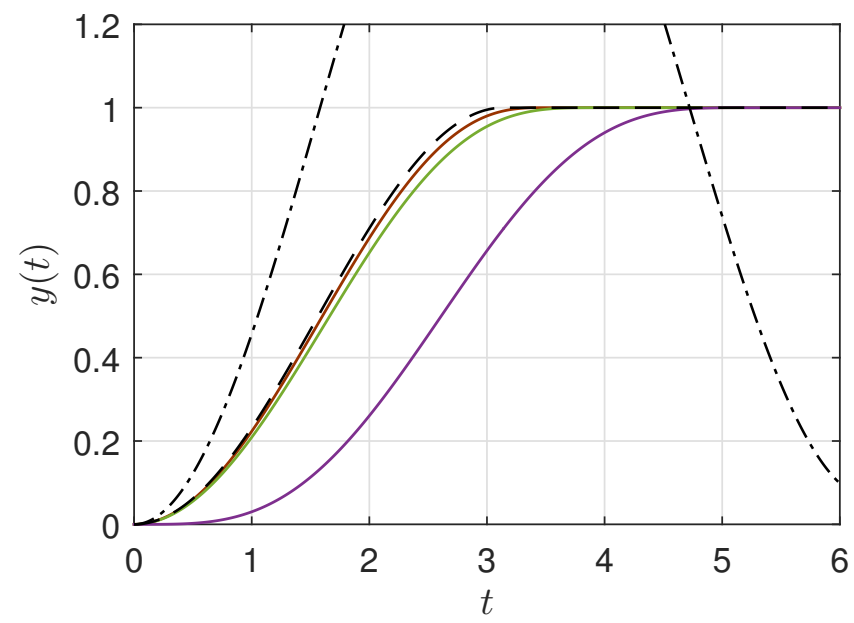

Figure 9: Step response of the shaper directly connected with a second order oscillatory system with output y for examples \#4(brown),\#5(green) and \#6(purple).

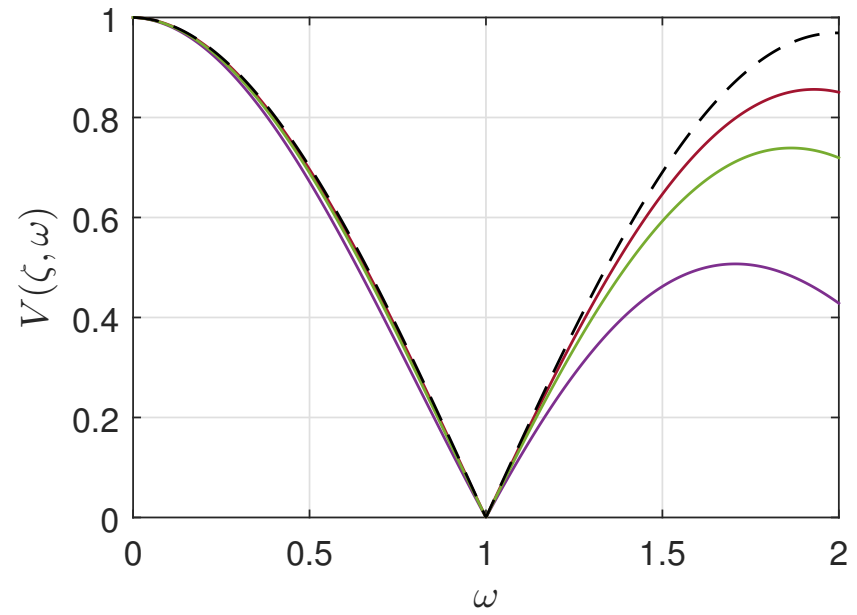

Figure 10: Residual vibrations of the shaper examples \#4(brown),\#5(green) and \#6(purple). The black dashed line corresponds to the ZV shaper. 


\subsection{Exponentially decaying basis functions}

The shaper described by (5) and (7) can be rewritten into the form

$$
y(t)=A u(t)+\int_{0}^{T} C e^{\mathbb{A} \theta} B u(t-\theta) d \theta,
$$

where

$$
\mathbb{A}=\left[\begin{array}{ccccc}
0 & 1 & 0 & \cdots & 0 \\
& 0 & \ddots & \ddots & \vdots \\
\vdots & & \ddots & \ddots & 0 \\
0 & & \cdots & 0 & 1
\end{array}\right] \in \mathbb{R}^{N_{p}+1 \times N_{p}+1}
$$

and

$$
B=\left[\begin{array}{llll}
0 ! a_{0} & 1 ! a_{1} & \ldots & N ! a_{N_{p}}
\end{array}\right]^{\top}, C=\left[\begin{array}{llll}
0 & \ldots & 0 & 1
\end{array}\right] .
$$

The corresponding transfer function can then also be expressed as

$$
G(s)=A+C(s I-\mathbb{A})^{-1}\left(I-e^{-T(s I-\mathbb{A})}\right) B .
$$

This suggest a realization and implementation by the dynamic system

$$
\left\{\begin{array}{l}
\dot{z}(t)=\mathbb{A} z(t)+B u(t)-e^{T \mathbb{A}} B u(t-T), \\
y(t)=C z(t)+A u(t) .
\end{array}\right.
$$

Unfortunately, the system $(39)$ is always unstable, because the matrix $\mathbb{A}$ has a multiple non-semisimple eigenvalue at zero. In (38), however, the eigenvalues of $\mathbb{A}$ are removable singularities. A general approach to approximate FIR filters by stable LTI systems is described in [56] and the references therein. In what follows we present a solution which does not involve an approximation.

The instability problem can be resolved by modifying the basis functions $g_{i}$. Given a number $\lambda>0$, one can also consider a delay kernel of the form

$$
g(\theta)=\sum_{i=0}^{N_{p}} a_{i} \theta^{i} e^{-\lambda \theta} .
$$

Note that the expression is still linear in $a_{i}$ so all results presented before regarding the tuning of the parameters $A, a_{0}, \ldots, a_{N_{p}}$ to compensate a zero, to have a non-negative step response, etc., can be easily adapted. In the implementation there is one difference. Namely, we still have

$$
g(\theta)=C e^{\mathbb{A} \theta} B
$$

with $B$ and $C$ as before but with

$$
\mathbb{A}=\left[\begin{array}{ccccc}
-\lambda & 1 & 0 & \cdots & 0 \\
& -\lambda & \ddots & \ddots & \vdots \\
\vdots & & \ddots & \ddots & 0 \\
& & & -\lambda & 1 \\
0 & & \cdots & & -\lambda
\end{array}\right] \in \mathbb{R}^{N_{p}+1 \times N_{p}+1}
$$




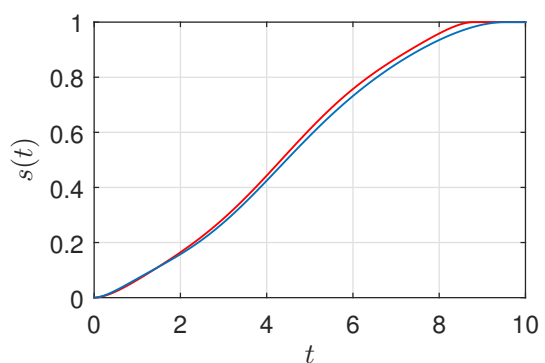

(a) Step responses

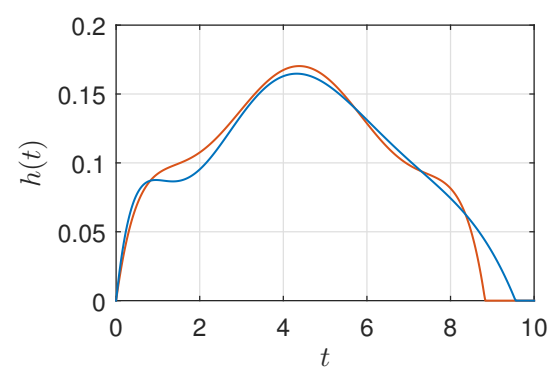

(b) Impulse responses

Figure 11: Step and impulse responses of the shaper examples with $\lambda=0$ (red) and $\lambda=0.4$ (blue)

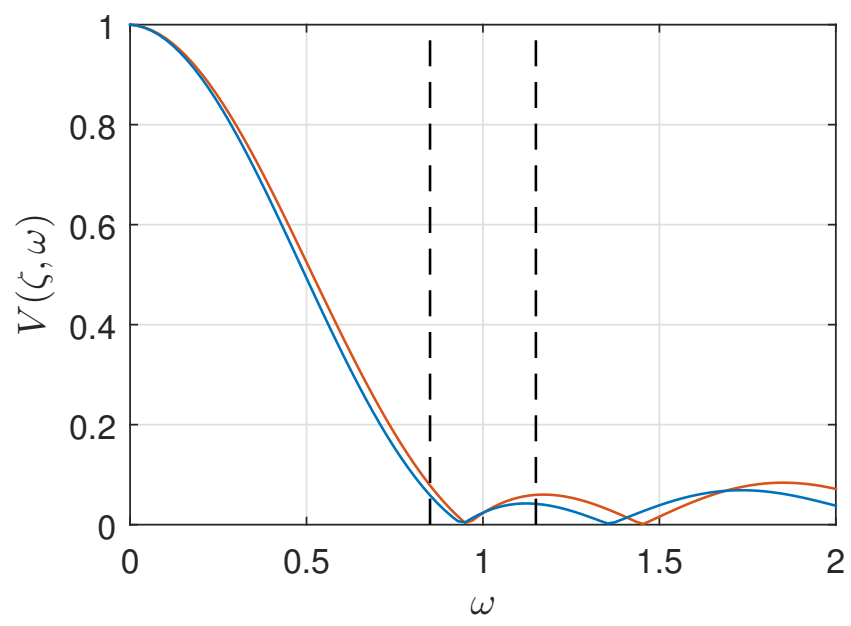

Figure 12: Residual vibrations of the shaper designed with $\lambda=0$ (red) and $\lambda=0.4$ (blue)

Now $\mathbb{A}$ is a Hurwitz matrix for $\lambda>0$, thus, the implementation using differential equation 39 is already stable.

The choice of $\lambda$, once again, induces a trade-off. For large $\lambda$, the additional dynamics are very fast and do not affect the overall control system's performance, but since the kernel basis functions have a rapid decay, it might become more difficult to find parameter values for the shaper satisfying the design requirements, affecting the performance.

Example. We revisit Example \#2 and compare the design for $\lambda=0$ and $\lambda=0.4$. A comparison of step and impulse response is shown in Fig. 11. where imposing $\lambda>0$ introduces a longer response time and a slight modification of the shape of the responses, see Fig. 12. The residual vibrations, see Fig. 12, are slightly different in the optimized interval with lower average residual vibrations. 


\section{Conclusions}

As the main contributions, a novel class of input shapers is proposed, as well as an integrated approach for designing its parameters. The main benefit compared to the previous work consists of relaxing the delay distribution from a fixed to a continuous (polynomial) shape along with a systematic design procedure grounded in convex optimization. Utilizing polynomial functions lead to major advantages. Firstly, the polynomial functions allow continuous derivatives of the step response which is not possible using discrete delays, see, e.g, examples \#2 and \#6. In comparison with distributed delay shapers with piecewise constant distribution proposed in [23, a smoother response is guaranteed at the starting and ending point of the response, and on top of that higher derivatives can also be limited. Secondly, the distributed delay has a retarded spectrum, which is an important requirement when the shapers are implemented as inverse shapers in a feedback look, see the spectra in Fig. 7. Finally, the input shapers with distributed delay mimic weighted moving averaging, which is an important feature when they are used in feedback where the measurement is under effect of noise.

The design of input shapers usually involves an important trade-off between performance, in terms of response time, and robustness. Instead of moving one of the objectives to a constraint, which is traditionally done and requires in practise a lot of iterations to appropriately define constraint levels, we address in this paper, for the first time, this trade-off by directly solving the design problem as a multi-objective optimization problem. The design of the parameters is based on convex optimization techniques, generating a set of Pareto optimal solutions. The decision maker can subsequently select the best fitting solution for his / her application. The degrees of freedom of the proposed method allow to introduce number of additional design options, such as limitation of the jerk (analogously to 24]) and jounce. Also continuity at the start and the end of the action can be prescribed. We also show scaling properties of the optimization problem. Let us remark that compared to the command smoothers considered in 22, when utilizing this option, the designed shaper/smoother may provide both command smoothing and full compensation of the mode. Additionally, next to the possibility to assess the robustness with respect to the frequency as it is common, the robustness with respect to the damping of the mode to be suppressed can be included as a requirement in the shaper design. The novelty is also in the option to include the overall action length of the shaper in the optimization procedure, next to the robustness of the mode suppression. The additional contributory aspect is the possible implementation of the shaper (time delay FIR filter) as a dynamical system with a discrete delay (39).

With regard to the generalized FIR filter designs in [41, the design method proposed in this paper utilize a different approach grounded in time delay system's theory and gives a more comprehensive design allowing to select parameters of the shaper that fit the particular application. Apart of that, the problem has not been solved in such complexity and variability of design options yet.

To facilitate the use of the approach, the paper is accompanied by a user- 
friendly publicly available design tool.

\section{Acknowledgments}

The presented research has been supported by the Czech Science Foundation under the project No. 16-17398S, by the Programme of Interuniversity Attraction Poles of the Belgian Federal Science Policy Office(IAP P6- DYSCO), by OPTEC, the Optimization in Engineering Center of the KU Leuven, and the project G.0712.11N and G.0717.11N of the Research Foundation - Flanders (FWO).

\section{References}

[1] O. J. Smith, Posicast control of damped oscillatory systems, Proceedings of the IRE 45 (9) (1957) 1249-1255.

[2] N. C. Singer, W. P. Seering, Preshaping command inputs to reduce system vibration, Journal of Dynamic Systems, Measurement, and Control 112 (1) (1990) 76-82.

[3] W. Singhose, W. Seering, N. Singer, Residual vibration reduction using vector diagrams to generate shaped inputs, Journal of Mechanical Design 116 (2) (1994) 654-659.

[4] W. Singhose, S. Derezinski, N. Singer, Extra-insensitive input shapers for controlling flexible spacecraft, Journal of Guidance, Control, and Dynamics 19 (2) (1996) 385-391.

[5] M. Schlegel, M. Goubej, Feature-based parametrization of input shaping filters with time delays, in: Time Delay Systems, Vol. 9, 2010, pp. 247-252.

[6] W. Singhose, E. Crain, W. Seering, Convolved and simultaneous two-mode input shapers, in: Control Theory and Applications, IEE Proceedings-, Vol. 144, IET, 1997, pp. 515-520.

[7] T. D. Tuttle, W. P. Seering, A zero-placement technique for designing shaped inputs to suppress multiple-mode vibration, in: American Control Conference, 1994, Vol. 3, IEEE, 1994, pp. 2533-2537.

[8] B. R. Murphy, I. Watanabe, Digital shaping filters for reducing machine vibration, Robotics and Automation, IEEE Transactions on 8 (2) (1992) 285-289.

[9] D. P. Magee, W. J. Book, Implementing modified command filtering to eliminate multiple modes of vibration, in: American Control Conference, 1993, IEEE, 1993, pp. 2700-2704.

[10] M. D. Baumgart, L. Y. Pao, Discrete time-optimal command shaping, Automatica 43 (8) (2007) 1403-1409. 
[11] T. Singh, S. Vadali, Robust time-delay control, Journal of dynamic systems, measurement, and control 115 (2A) (1993) 303-306.

[12] S. Gürleyük, Ş. Cinal, Robust three-impulse sequence input shaper design, Journal of Vibration and Control 13 (12) (2007) 1807-1818.

[13] J. Vaughan, A. Yano, W. Singhose, Comparison of robust input shapers, Journal of Sound and Vibration 315 (4) (2008) 797-815.

[14] K. A. Alghanim, K. A. Alhazza, Z. N. Masoud, Discrete-time command profile for simultaneous travel and hoist maneuvers of overhead cranes, Journal of Sound and Vibration 345 (2015) 47-57.

[15] Z. Hurák, M. Hromčík, M. Špiller, Minimization of 2 norm of the error signal in posicast input command shaping: a polynomial approach, International Journal of Robust and Nonlinear Control 17 (8) (2007) 706-719.

[16] D. Kim, W. Singhose, Performance studies of human operators driving double-pendulum bridge cranes, Control Engineering Practice 18 (6) (2010) $567-576$.

[17] Z. Mohamed, J. Martins, M. Tokhi, J. S. Da Costa, M. Botto, Vibration control of a very flexible manipulator system, Control Engineering Practice 13 (3) (2005) 267-277.

[18] X. Yang, J. Cui, D. Lao, D. Li, J. Chen, Input shaping enhanced active disturbance rejection control for a twin rotor multi-input multi-output system (trms), ISA transactions 62 (2016) 287-298.

[19] E. Pereira, J. R. Trapero, I. M. Díaz, V. Feliu, Adaptive input shaping for manoeuvring flexible structures using an algebraic identification technique, Automatica 45 (4) (2009) 1046-1051.

[20] J. Park, P.-H. Chang, H.-S. Park, S. Lee, Design of learning input shaping technique for residual vibration suppression in an industrial robot, Mechatronics, IEEE/ASME Transactions on 11 (1) (2006) 55-65.

[21] P. H. Meckl, P. B. Arestides, Optimized s-curve motion profiles for minimum residual vibration, in: American Control Conference, 1998. Proceedings of the 1998, Vol. 5, IEEE, 1998, pp. 2627-2631.

[22] W. Singhose, R. Eloundou, J. Lawrence, Command generation for flexible systems by input shaping and command smoothing, Journal of guidance, control, and dynamics 33 (6) (2010) 1697-1707.

[23] T. Vyhlídal, Hromčík, Parameterization of zero vibration shapers with delays of various distribution, Automatica 59 (2015) 256-263.

[24] T. Singh, Jerk limited input shapers, in: American Control Conference, 2004. Proceedings of the 2004, Vol. 5, IEEE, 2004, pp. 4825-4830. 
[25] T. Vyhlídal, V. Kučera, M. Hromčík, Input shapers with uniformly distributed delays, in: In Proc. of 10th IFAC workshop on time delay systems, Vol. 10, Time delay systems, 2012, pp. 91-96.

[26] T. Vyhlídal, V. Kučera, M. Hromčík, Signal shaper with a distributed delay: Spectral analysis and design, Automatica 49 (11) (2013) 3484-3489.

[27] T. Vyhlídal, V. Kučera, M. Hromčík, Zero vibration shapers with distributed delays of various types, in: Decision and Control (CDC), 2013 IEEE 52nd Annual Conference on, IEEE, 2013, pp. 940-945.

[28] T. Vyhlídal, M. Hromčík, V. Kučera, Inverse signal shapers in effective feedback architecture, in: Control Conference (ECC), 2013 European, IEEE, 2013, pp. 4418-4423.

[29] T. Vyhlídal, M. Hromčík, V. Kučera, M. Anderle, On feedback architectures with signal shapers, IEEE Transactions on Automatic Control.

[30] J. K. Hale, S. M. V. Lunel, Introduction to functional differential equations, Vol. 99, Springer Science \& Business Media, 2013.

[31] J. K. Hale, S. M. V. Lunel, Strong stabilization of neutral functional differential equations, IMA Journal of Mathematical Control and Information 19 (1 and 2) (2002) 5-23.

[32] J. K. Hale, S. V. Lunel, Stability and control of feedback systems with time delays, International Journal of Systems Science 34 (8-9) (2003) 497-504.

[33] M. J. Robertson, W. E. Singhose, Multi-level optimization techniques for designing digital input shapers, in: American Control Conference, 2001. Proceedings of the 2001, Vol. 1, IEEE, 2001, pp. 269-274.

[34] L. Van den Broeck, G. Pipeleers, J. De Caigny, B. Demeulenaere, J. Swevers, J. De Schutter, Inputshaping: a linear programming approach, in: Proceedings of the International Conference on Noise and Vibration Engineering, 2008, pp. 397-408.

[35] M. Goubej, Robust motion control of flexible electromechanical systems, PhD Thesis supervised by M. Schlegel, Faculty of applied sciences, University of West Bohemia, 2014.

[36] K. A. Alhazza, Z. N. Masoud, Waveform command shaping control of multimode systems, Journal of Sound and Vibration 363 (2016) 126-140.

[37] D.-W. Peng, T. Singh, M. Milano, Zero-phase velocity tracking of vibratory systems, Control Engineering Practice 40 (2015) 93-101.

[38] R. van der Maas, T. Singh, M. Steinbuch, Application of a time-delay prefilter design for periodic signal tracking of lightly-damped multivariable systems, IFAC-PapersOnLine 48 (12) (2015) 135-140. 
[39] M. O. Cole, A class of low-pass fir input shaping filters achieving exact residual vibration cancelation, Automatica 48 (9) (2012) 2377-2380.

[40] M. O. Cole, T. Wongratanaphisan, A direct method of adaptive fir input shaping for motion control with zero residual vibration, Mechatronics, IEEE/ASME Transactions on 18 (1) (2013) 316-327.

[41] M. O. Cole, T. Wongratanaphisan, Optimal fir input shaper designs for motion control with zero residual vibration, Journal of Dynamic Systems, Measurement, and Control 133 (2) (2011) 021008.

[42] W. E. Singhose, L. J. Porter, T. D. Tuttle, N. C. Singer, Vibration reduction using multi-hump input shapers, Journal of dynamic systems, Measurement, and control 119 (2) (1997) 320-326.

[43] W. Fan, X.-S. Gao, W. Yan, C.-M. Yuan, Interpolation of parametric cnc machining path under confined jounce, The International Journal of Advanced Manufacturing Technology 62 (5-8) (2012) 719-739.

[44] S.-H. Nam, M.-Y. Yang, A study on a generalized parametric interpolator with real-time jerk-limited acceleration, Computer-Aided Design 36 (1) (2004) $27-36$.

[45] X. Broquere, D. Sidobre, K. Nguyen, From motion planning to trajectory control with bounded jerk for service manipulator robots, in: Robotics and Automation (ICRA), 2010 IEEE International Conference on, 2010, pp. $4505-4510$.

[46] S. Macfarlane, E. Croft, Jerk-bounded manipulator trajectory planning: design for real-time applications, Robotics and Automation, IEEE Transactions on 19 (1) (2003) 42-52.

[47] T. Besselmann, Constrained optimal control, Diss., Eidgenössische Technische Hochschule ETH Zürich, Nr. 18950, 2010, 2010.

[48] G. H. Hardy, J. E. Littlewood, G. Pólya, Inequalities, Cambridge university press, 1952.

[49] W. Singhose, W. Seering, N. Singer, Residual vibration reduction using vector diagrams to generate shaped inputs, Journal of Mechanical Design 116 (2) (1994) 654-659.

[50] G. Stewart, Afternotes on numerical analysis, Society for Industrial and Applied Mathematics, Philadelphia, c1996.

[51] K. Miettinen, Nonlinear multiobjective optimization, Vol. 12, Springer Science \& Business Media, 2012.

[52] C.-L. Hwang, A. S. M. Masud, Multiple objective decision makingmethods and applications: a state-of-the-art survey, Vol. 164, Springer Science \& Business Media, 2012. 
[53] S. Boyd, L. Vandenberghe, Convex optimization, Cambridge university press, 2004.

[54] T. Vyhlídal, P. Zítek, Qpmr - quasi-polynomial rootfinder: algorithm and examples, in Delay Systems: from Theory to Numerics and Applications, Advances in Delays and Dynamics 1.

[55] S. Mondié, W. Michiels, Finite spectrum assignment of unstable time-delay systems with a safe implementation, IEEE Transactions on Automatic Control 48 (12) (2003) 2207-2212.

[56] W. Michiels, H. U. Unal, Evaluating and approximating fir filters: an approach based on functions of matrices, Automatic Control, IEEE Transactions on 60 (2) (2015) 463-468. 